\title{
Philosophiques
}

\section{La dynamique de Leibniz : métaphysique et substantialisme François Duchesneau, La dynamique de Leibniz, Paris, Vrin, coll. Mathesis, 1994}

\section{Yves Gingras}

Volume 22, numéro 2, automne 1995

URI : https://id.erudit.org/iderudit/027343ar

DOI : https://doi.org/10.7202/027343ar

Aller au sommaire du numéro

Éditeur(s)

Société de philosophie du Québec

ISSN

0316-2923 (imprimé)

1492-1391 (numérique)

Découvrir la revue

Citer cet article

Gingras, Y. (1995). La dynamique de Leibniz : métaphysique et substantialisme

/ François Duchesneau, La dynamique de Leibniz, Paris, Vrin, coll. Mathesis,

1994. Philosophiques, 22(2), 395-405. https://doi.org/10.7202/027343ar d'utilisation que vous pouvez consulter en ligne.

https://apropos.erudit.org/fr/usagers/politique-dutilisation/ 


\title{
LA DYNAMIQUE DE LEBNIZ : MÉTAPHYSIQUE ET SUBSTANTIALISME
}

\author{
par Yves Gingras
}

Depuis la publication en 1934 du livre de Martial Gueroult, Leibniz. Dynamique et métaphysique, le domaine des études leibniziennes a été profondément renouvelé par la multiplication des études et par la découverte de nouveaux manuscrits". En 1956, Pierre Costabel mettait au jour l'Essay de dynamique de $1692^{1}$. Quinze ans plus tard Michel Fichant faisait connaître un inédit de grande importance, le De Corporum Concursu de 1678, qui modifiait de façon importante la chronologie recue de l'évolution de la pensée du bibliothécaire de Hanovre ${ }^{2}$. Depuis le début des années 1970 la publication de la revue Studia Leibnitiana a aussi beaucoup contribué à ce qui semble bien être un " renouveau des études leibniziennes » en stimulant l'étude de multiples aspects de la pensée du philosophe.

En publiant La dynamique de Leibniz, François Duchesneau, tire profit de l'ensemble de ces travaux et propose une nouvelle synthèse, mais aussi une analyse renouvelée, du cheminement conceptuel de Leibniz dans ce domaine particulier de la physique. D'une grande érudition, l'étude de Duchesneau est aussi toute en nuance et il faut bien avouer que seuls les véritables Leibniz scholars, comme on dit si bien en anglais, poumont évaluer à leur juste mesure les apports de l'auteur à ce secteur de l'histoire de la philosophie. Chose certaine, et cela dit sans céder en rien aux formules toutes faites, l'étude serrée de tous les textes importants qui jalonnent le parcours de la pensée dynamique de Leibniz fait de ce livre une référence incontournable pour quiconque s'intéresse à l'histoire de l'épistémologie ou des sciences au siècle de la révolution scientifique.

Mes préoccupations étant celles d'un historien de la physique et non d'un exégète de la pensée de Leibniz, et ayant toujours cru que le plus bel hommage qu'un chercheur puisse rendre à un auteur soit d'intégrer ses travaux à ses propres réflexions, mes commentaires se borneront donc à soulever des questions relatives aux contributions du philosophe allemand à la conceptualisation de la physique. Car pour tout dire, je ne pouvais refuser, tout néophyte

* M. Gueroult, Leibniz. Dynamique et métaphyrique, Paris, Aubier-Montaigne, 1967 (1ère édition 1934).

1. P. Costahel, Leibniz et la dynamique en 1692. Textes et commentaires, Paris, Vrin 1981 (1ère édition 1960).

2. G.W. Leihniz, La réfome de la dynamique, textes inédits, traduits et commentés par M. Fichant, Paris, Vrin 1994 
que je fusse en matière leibnizienne, cette occasion qui m'était offerte de soumettre à un érudit de son calibre certaines thèses qui me tiennent à cœur et que j'ai aujourd'hui la témérité de lui soumettre en toute cordialité ; et ce, même si ma présentation n'a rien de la diplomatie avec laquelle il aborde l'étude de Gueroult qui, plus d'un demi-siècle après sa publication, demeure un phare sur lequel les spécialistes de Leibniz continuent de se positionner même si sa lueur s'est quelque peu affaiblie. Bien que plus physicien que philosophique, mon parcours m'amènera tout de même à soulever des questions relatives à la « méthode de la science » qui est au çeur des préoccupations de Duchesneau.

\section{Physique et dynamique}

Malgré la référence à la dynamique dans le titre, on peut dire que l'ouvrage de Duchesneau est davantage une contribution à l'histoire de la philosophie et de la philosophie des sciences qu'à celle de la physique. L'auteur se concentre en effet sur l'évolution des " styles épistémologiques qui se développent successivement dans les textes préparant, puis consacrant la dynamique ${ }^{3}$ ". La méthode adoptée est celle, classique, de l'analyse interne des textes qui permet de "suivre la genèse de la dynamique comme science [...] dans la structure argumentative des textes leibniziens ${ }^{4}$ ". L'ouvrage est d'ailleurs un complément naturel à son Leibniz et la méthode de la science paru un an plus tôt.

Pour ce qui concerne les questions de physique proprement dite, on peut d'ailleurs se surprendre de trouver le traitement du Tentamen de motuum colestium causis, un texte qui est au cceur de la pensée physique de Leibniz, dans le livre consacré à la méthode plutôt que dans celui dédié à la dynamiqué. Aussi, les concepts relevant de la physique (masse, densité, inertie, éther) ne font pas vraiment l'objet d'une analyse conceptuelle à laquelle un historien de la physique aurait pu s'attendre, en ouvrant un livre intitulé "La dynamique de Leibniz". Il est entendu que la présence des termes "masse" ou "densité » n'est pas suffisante pour attester la présence d'un concept bien formé. Pour ne donner qu'un exemple, mentionnons l'impossibilité dans le cadre pléniste de formuler clairement le concept de densité, à moins d'invoquer autant de matières distinctes qu'il y a de degrés de densité. Cette difficulté mine d'ailleurs la consistance interne de tous les modèles plénistes (de Descartes à Leibniz inclus), qui invoquent des fluides «plus ou moins subtils ». En fait, le concept de densité ne sera clairement formulé que dans un cadre qui fait place au vide. La question de l'inertie naturelle des corps demanderait aussi un traitement approfondi.

3. F. Duchesneau, La dynamique de Leibniz, Paris, Vrin, 1994, p. 11.

4. Ibid, p. 12-13; les soulignés sont de nous

5. F. Duchesneau, Leibniz et la méthode de la science, Paris, PUF, 1993, p. 229-258. 
L'auteur ayant choisi de déployer son analyse sur le versant méthodologique de la pensée de Leibniz, on ne saurait toutefois lui faire grief de ces silences, mais il faut avouer que ce parti pris, tout légitime qu'il soit, résulte en un traitement incomplet de la physique de Leibniz. A moins bien sûr de ne retenir du terme «dynamique » que son acception leibnizienne stricte qui l'identifie à la force vive (vis viva). Mais cela aurait encore le defaut de dissocier artificiellement la physique de la dynamique.

Comme le notait avec justesse René Dugas, « pour suivre la pensée mécanique de Leibniz, il faut tout d'abord avoir entendu les grands mécaniciens du siècle et les garder constamment à l'esprit ${ }^{6} »$. Or, il me semble que l'analyse interne des textes ne permet pas toujours de foumir les repères essentiels.

Pour bien comprendre la spécificité de la pensée physique de Leibniz, il faut le replacer dans le petit monde de la physique de l'époque et surtout rappeler qu'au delà de l'accord assez général sur la nécessité de l'explication mécanique de la nature, deux paradigmes s'affrontent alors : celui du plein, héritier de Descartes, et celui de l'atomisme et du vide, dans la tradition de Gassendi et Galilée repris par les savants britanniques réunis autour de la Royal Society de Londres. Ces caractéristiques centrales du projet physique leibnizien me semblent insuffisamment situées en rapport avec les contributions des principaux savants de l'époque, ce qui a pour effet de magnifier son originalité conceptuelle.

Lorsqu'il fait son entrée dans le monde de la physique en publiant à l'âge de vingt-cinq ans la Theoria motus abstracti (dédiée à l'Académie des sciences de Paris) et la Theoria motus concreti (ou Hypothesis physica nova) (dédiée pour sa part à la Royal Society de Londres), Leibniz se situe d'emblée du coté des plénistes. Du point de vue physique, sa théorie du mouvement concret, en particulier, ne fait qu' ajouter un modèle tourbillonnaire de plus à ceux qui existaient déjà. Après l'essai fondateur de Descartes dans ses Principia Philosophia, il y eut, entre autres, ceux de Christian Huygens, présenté à l'Académie des sciences en 1669 lors d'une séance sur les causes de la pesanteur (mais publié en 1690 en appendice à son Traité de la lumière), et du savant italien Giovanni Alphonso Borelli qui publia trois ans plus tôt sa Theorica medicearum planetarum ex causis physicis deducta ${ }^{7}$. Bien que Michel Blay ait raison de noter l'analogie entre cet essai et celui de Leibniz (les deux auteurs utilisant trois forces de même nature pour expliquer le mouvement elliptique des planetes) ${ }^{8}$, il n'est nul besoin d'invoquer une quelconque influence de l'un sur l'autre pour expliquer ces ressemblances qui sont en fait des convergences. Il suffit en effet de noter que dans une conceptualisation

6. R. Dugas, La mécanique au XVIIe siècle, Neuchatel, Éditions du Griffon, 1954, p. 461 .

7. Sur la question des tourbillons en général, voir E. I. Ayton, The Vortex Theory of Planetary Motion, New York, American Elsevier, 1972

8. M. Blay, La naissance de la mécanique analytique. Paris, PUF, 1992, p. 142. 
pléniste et mécaniste du mouvement, la pesanteur ne peut s'expliquer que par l'action d'un fluide plus ou moins subtil, ou d'une combinaison de tels fluides. En fait c'est là aussi le mécanisme explicatif de base pour toute conception du mouvement en termes d'impulsions et de forces de contact.

Anxieux de connaître l'avis des maitres anglais sur ses deux premiers essais consacrés à la théorie du mouvement, le jeune Leibniz demande à Oldenburg de lui transmettre si possible leur opinion, surtout celle de Hooke, Boyle, Wren et Wallis. À sa séance du 4 mai, la Société confia à Hooke le soin de faire rapport sur l' Hypothesis physica nova. Ce dernier s'exécute à la séance suivante (11 mai), se disant simplement dissatisfied with it ${ }^{9}$. Quant au Theoria motus abstracti, il croit que Leibniz had not hit right $t^{10}$ et la Société référa le texte à Wallis. Ce dernier avait fait connaître sa réaction à la Theoria motus concret $i$ à Oldenburg dans une lettre du 7 avril. Il y constatait l'accord sur plusieurs points entre les explications de Leibniz et les siennes propres avancées dans ses Mechanica sive de motu, parues l'année précédente. Sur la gravitation, il affirmait ses convictions mécanistes à l'effet que although the cause of gravity (and elasticity too) is so very obscure that I also have never yet been satisfied as to what conclusion I should reach concerning it, still, the phenomena of nature are for the most part better explained by pressures than by attraction. Sur la question de la cohésion de la matière, point sur lequel la théorie de Leibniz est opposée à celle de Boyle (le premier l'expliquant par le mouvement, le second par le repos), il se contentait de dire que the question must be left to time and the arguments of the learned on either side ${ }^{11}$. Wallis sera moins disert sur la Theoria motus abstracti, avouant que « it may seems invidious to criticize the writings of others $»$. Il note tout de même son accord sur plusieurs points, mais doute de l'explication de la cohésion avancée par Leibniz, qui est d'ailleurs similaire à celle formulée par William Neale en 1667. En somme, il ne veut pas prononcer d'opinions préjudiciables aux autres so that everyone may freely adopt whatever view shall seem in his judgement most agreable to reason ${ }^{12}$.

Lorsque Oldenburg fera parvenir ces opinions à Leibniz, il prendra soin de préparer le philosophe à les recevoir en lui rappelant que philosophers diverge into a variety of opinions [...] about matters lying outside the rigours of mathematics. Passant outre à l'opinion peut-être trop lapidaire de Hooke, il

9. T. Birch, The History of th Royal Society of London, London, 1756, (reprint 1967), vol. 2, p. 479 et 481 .

10 Ibid, p. 482.

11. Wallis à Oldenburg, 7 avril 1671, in The Correspondence of Henry Oldenburg, Madison, The University of Wisconsin Press, 1971, vol. 7, p. 562-563.

12. Wallis à Oldenhurg, 2 juin 1671 , in The Correspondence of Henry Oldenburg, Madison, The University of Wisconsin Press, 1971, vol. 8, p.73; voir aussi sa lettre du 6 novembre 1671, idem, p. 342-343. 
résume la réception de ses æuvres en notant que he who seemed most of all to favor your views was the famous Wallis ${ }^{13}$.

Tout bien considéré, il me semble qu' on est loin de ce que Michel Fichant considère comme un accueil « plutôt favorable ${ }^{14}$ ». Les philosophes britanniques de la nature semblent plutôt avoir réagi poliment, c'est-à-dire en bons "gentlemen ", à ce qu'ils ne pouvaient manquer de percevoir comme une voie a priori, qui était aux antipodes de leurs idées sur la production de matters of fact.

Intéressé par la méthodologie de Leibniz davantage que par sa pensée physique, Duchesneau a probablement raison de « tenir le projet de 1671 pour profondément original dans son orientation méthodologique " et de déplorer que Mouy et Dugas aient eu tendance à sous-estimer la différence entre Leibniz et Descartes sur ce point. Cependant, il est possible que cette différence d'appréciation provienne du fait que le point de vue adopté par ces auteurs est celui d'historiens de la physique alors que Duchesneau adopte le regard de l'historien de la philosophie (ou, plus précisément, de l'épistémologie). Car au-delà des différences de consuructions effectives (rejet des corpuscules cartésiens par Leibniz, par exemple), c'est bien le paradigme pléniste qui l'unit fondamentalement à Descartes sur le plan physique et qui l'opposera toujours aux physiciens anglais et à Newton en particulier. Comme le note l'auteur, "l'hypothèse de l'éther subsistera dans la pensée physique ultérieure : il n'y aura pas de véritable renonciation aux arguments de la Theoria motus concreti, alors même que la Theoria motus abstracti aura été remplacée par les principes de la dynamique ${ }^{15}$ ". Et cela parce que l'auteur n'abandonnera jamais les principes a priori de continuité et de contiguïté des interactions (impulsions).

\section{Leibniz : l'« inventeur » de la dynamique ?}

Dans l'histoire de la physique, le nom de Leibniz est essentiellement associé à la controverse des forces vives qui occupa le monde savant pendant plus d'un demi siècle (disons de 1685 à 1750). Il me semble que, sur cette question encore, l'élude purement interne des textes de Leibniz a pour effet de trop accorder à l' inventeur du mot "dynamique " (et non du domaine de recherche qui consiste à étudier les forces agissant sur les corps et qui en astronomie remonte à Kepler). Ainsi, l'analyse minutieuse de la démarche de Leibniz dans le De Corporum Concursu de 1678, tend à laisser dans l'ombre le fait, à mes yeux fondamental, que le philosophe reprend intégralement les équations de Huygens et n'ajoute qu'une vision substantialiste à l'équation de la conservation de la somme des carrés des vitesses, établie par Huygens dans

13. Oldenburg à Leibniz, 12 juin 1671 , in Ibid, vol. 8, p. 103.

14. M. Fichant, «Introduction » à G.W. Leibniz, La réforme de la dynamique, textes inédits, op. cit., p. 35.

15. F. Duchesneau, La dynanique de Leibniz, op. cit., p. 67. 
son essai sur le choc des corps de 1669 . On peut comprendre la tendance des auteurs de l'époque à ne pas trop insister sur leurs emprunts même lorsqu'ils sont massifs, mais l'analyste ne doit-il pas garder ses distances ? Or «l'inventeur de la dynamique », pour reprendre une expression de Duchesneau, réinterprète dans un cadre ontologique les lois que Huygens avait codifié mathématiquement. C'est surtout cela qui sépare le philosophe du savant. Notons que l'idée qu'une quantité conservée suggère l'existence d'une substance sous-jacente sera souvent reprise. On a qu'à penser à la loi de continuité en mécanique des fluides, que l'on retrouve aussi en électricité et qui suggère l'existence d'un fluide électrique. On peut bien sûr observer le cas contraire : le postulat de l'existence du fluide calorique amène naturellement à écrire une loi de conservation du calorique.

La substantialisation de la notion de force vive, qui fait de la force une entité active au sein même de la masse, éloigne d'ailleurs radicalement Leibniz d'une notion de force conçue, chez Newton entre autres, comme externe à l'objet, notion d'ailleurs inséparable d'une conception de l'inertie comme tendance à persister dans l'état de mouvement. Il faudrait aussi soulever la question, trop complexe pour être vraiment abordée ici, de la conceptualisation de la masse, qui fait défaut tant chez Descartes que chez Leibniz. On sait en effet que Emst Mach niait que la physique de Leibniz fit place au concept de masse alors que Couturat lui en attribuait la paternite $e^{16}$. Reprenant la question, Max Jammer, conclut plutôt que celle qui s'énonce dans le Dynamica de potentia de 1689 est toute newtonienne, bien qu'à mon sens il sous estime le fait que là ou Newton parle de " corps », Leibniz parle de « corps en mouvement », nuance qui a peut-être son importance ${ }^{17}$. Quoi qu'il en soit, il est probable que la difficulté à concevoir la notion de masse ait quelque chose à voir avec la notion d'inertie et de plein.

Ainsi, parler de Leibniz comme "l'inventeur de la dynamique " peut porter à confusion. Bien qu'il lance le terme, il n'a pas le monopole de celui de « force » (vis), et ce d'autant moins que la « dynamique» (au sens large) qui finira par s'imposer est celle de Newton. Pour bien apprécier la physique de Leibniz, il faudrait aussi rappeler tout ce que le philosophe doit à Kepler ${ }^{18}$. Tout compte fait, il serait peut-être plus juste, bien qu'encore là insatisfaisant, de parler de l'inventeur de la «force vive " (vis viva).

\section{Le spectre de Newton}

Mais ce qui frappe sans doute le plus dans la façon dont Duchesneau aborde la dynamique de Leibniz est l'absence d'une véritable confrontation

16. E. Mach, The Science of mechanics, La Salle, Open Court, 1960, p. 365.

17. M. Jammer, Concepts of Mass in Classical and Modern Physics, Harper Torchbooks, p. 76 et 79 ; L. Couturat, « Le système de Leibniz d'après M. Cassirer », Revue de métaphysique et de morale, vol. 11, 1903, p. 89.

18. M. Jammer, op. cit., p. 79. 
avec Newton, qui, rappelons-le, publie ses Philosophice naturalis principia mathematica, en 1687. Il est d'ailleurs symptomatique que l'édition de Fellman des notes marginales de Leibniz à sa copie du livre de Newton ne figure dans la bibliographie d'aucun de ses deux volumes ${ }^{19}$. Il ne s'agit pas bien sûr de mettre à l'épreuve l'érudition de l'auteur, mais plutôt de suggérer que le traitement strictement interne d'une auvre a ses limites, surtout lorsqu'elle interdit une mise en contexte adéquate avec les æuvres contemporaines avec lesquelles celles de Leibniz étaient en compétition, et par rapport auxquelles elles se démarquaient.

Or, la confrontation entre Newton et Leibniz est cruciale pour faire ressortir non seulement la spécificité de la physique leibnizienne mais aussi comprendre pourquoi elle n'a pas eu de suite. Car on ne peut sérieusement invoquer un putatif "éloignement progressif du paradigme newtonien ${ }^{20}$ " ou encore une hypothétique affinité de la « science actuelle [...] avec un programme qui inscrivait la construction des modeles " géométriques" sous des principes régulateurs ${ }^{21}$ » pour justifier un retour à la conception leibnizienne de l'explication physique. Soyons clair : par delà les limites aujourd'hui évidentes de la mécanique newtonienne (révélées par la relativité et la physique quantique), l'essentiel du «paradigme newtonien », par rapport à un éventuel « paradigme leibnizien » est toujours bien en place car il consiste justement en une mathématisation complète de la nature, laquelle se fait d'ailleurs au détriment des explications mécanistes et substantialistes. En d'autres termes, loin de participer à la mechanization of the world picture comme le suggère le titre célèbre du livre de Dijksterhuis, Newton inaugure une démécanisation par le biais de la mathématisation ${ }^{22}$. En somme, et pour reprendre les termes de Cassirer, Newton remplace la substance par la fonction, ce que n'a jamais pu (ni voulu) faire Leibniz ${ }^{23}$.

J'avoue donc ne pas très bien comprendre ce que veux dire Duchesneau lorsqu'il réfere à " des savoirs scientifiques qui renoncent de plus en plus au modèle newtonien » et qu'il suggère que ce dernier n'est « jamais totalement parvenu » à supplanter la méthodologie leibnizienne ${ }^{24}$. Je ne vois pas non plus "la parenté par rapport à la méthodologie contemporaine" de "l'approche explicative par recours à des principes architectoniques [...] dans un contexte d'analyse des phénomènes ${ }^{25}$ ". À moins bien sûr de s'en tenir uniquement à l'approche hypothético-déductive ou de vouloir projeter artificiellement dans

19. G.W. Leibniz, Marginalia in Newtoni Principia Mathematica (1687), édité par E. A. Fellman, Paris, Vrin, 1971.

20. F. Duchesneau, La dynamique de Leibniz, op. cit., p. 7.

21. Ibid, p. 341.

22. E. J. Dijksterhuis, The Mechanization of the World Picture, Princeton, Princeton University Press, 1986.

23. E. Cassirer, Substance et fonction, Paris, Minuit, 1977.

24. F. Duchesneau, Leibniz et la méthode de la science, op. cit., p. 11.

25. lbid., p. 8. 
la physique du champ ou la théorie de la relativité les principes métaphysiques et architectoniques leibniziens. Chose certaine, trois siecles d'histoire de la physique mettent clairement en évidence une « vection », comme disait Piaget, soit l'abandon progressif de toutes les substances invoquées pour rendre compte mécaniquement des phénomènes : tourbillon, fluide électrique, éther, masse, lesquelles ont été remplacées par des systèmes d'équations formelles.

L'opposition entre substantialisme et fonctionnalisme est également visible dans la correspondance de Leibniz avec Huygens sur la question de l'explication du mouvement céleste et de la conceptualisation de la force vive. En somme, on ne peut faire l'économie d'une étude comparée du Tentamen et des Principia, car c'est, à notre avis, à ce niveau que l'on voit le mieux les limites de la pensée mécaniste et substantialiste de Leibniz et que l'on comprend pourquoi elle n'a pas eu de suite.

Si nous nous accordons avec Duchesneau pour admettre que Leibniz est le «promoteur d'une conception originale » de la méthode, on aura compris que nous ne croyons pas toutefois qu'elle soit pour autant «à maints égards actuelle $^{26}$ ».

\section{Les mathématiques comme acide de la substance}

Il fait peu de doutes que Leibniz était fier d'avoir démontré que les lois de Kepler peuvent s'expliquer par l'existence de son tourbillon harmonique (qui postule une relation de la forme $v=c / r$ ), alors même que Newton, dans ses fameuses propositions LI et LII à la fin du livre II des Principia prétendait démontrer mathématiquement l'inexistence des tourbillons cartésiens. À cet égard, il n'est pas étonnant que parmi les annotations de Leibniz en marge des Principia, le plus grand nombre se retrouve autour des propositions portant sur les relations entre la force centripète et la forme elliptique de la trajectoire des planètes, les autres indiquant l'attention portée par le philosophe aux propositions de Newton concernant le mouvement d'un corps dans un fluide résistant (incluant la proposition $\mathrm{LI})^{27}$.

Comme le rappelle l'auteur, Leibniz a préparé le Tentamen en réaction à la lecture d'un long compte rendu des Principia paru dans les Acta Eruditorum de juin 1688. Leibniz ne semble toutefois pas avoir réalisé que la loi des aires n'est pas seulement la conséquence d'un tourbillon harmonique mais bien de toute force centrale comme l'a démontré Newton (Principia, Livre I, section II, propositions 1 et 2). De plus, pour expliquer la gravitation Leibniz invoque, en plus du tourbillon qui génère la force centrifuge, un deuxième

26. F. Duchesneau, La dynamique de Leibniz, op. cit., p. 7 ; le souligné est de nous.

27. Voir G.W. Leibniz, Marginalia in Newtoni Principia Mathematica (1687), op. cit. Rappelons qu'en plus du Tentamen Leibniz publia au même moment une étude sur le mouvement dans un fluide résistant, publication également stimulée par la lecture du compte-rendu des Principia. 
éther conçu en analogie avec la lumière et dont l'intensité diminue comme le carré de la distance. Or, Huygens lui fait justement remarquer qu'il est curieux qu'il doive invoquer trois forces pour rendre compte de ce que Newton explique avec seulement deux forces. Invoquer ainsi les "tourbillons de Descartes " - comme les appelle Huygens avec une certaine ironie - lui paraît superflu ${ }^{28}$. Il aurait peut-être pu ajouter que cette différence tient à une notion ambiguë d'inertie chez Leibniz. En effet, chez Newton c'est la force centripète d'attraction qui équilibre la tendance centrifuge due à l'inertie du corps, alors que chez Leibniz, c'est le tourbillon qui génère la force centrifuge, comme si l'inertie naturelle du corps n'y suffisait pas, ou n'existait tout simplement pas. À cela s'ajoute nombre de difficultés techniques notées par Huygens (trajectoire des comètes, accélérations et décélérations des planètes $^{29}$ ), obstacles qui demeureront insurmontés et qui étaient sans doute insurmontables, tant ils nécessitent la multiplication d'hypothèses ad hoc. Si la nouvelle version du Tentamen « restera malheureusement inédite » comme le déplore Duchesneau ${ }^{30}$, c'est peut-être que Leibniz, qui a pris le temps d'annoter sa propre copie des Principia et a été sensible aux commentaires de Huygens, a réalisé tout ce que son modèle comportait d'insatisfaisant.

Face aux tentatives infructueuses d'explication mécanique, on peut concevoir l'émergence de la notion d'action à distance comme une conséquence directe de la mathématisation de la physique. Ainsi que semble l'avoir saisi confusément Newton, la physique se heurte en quelque sorte à la mathématique et entre les deux il choisit la seconde quitte à transformer ainsi la nature même du travail du physicien.

La fameuse remarque dans le compte rendu des Principia paru dans le Journal des sçavans en août 1688 à l'effet qu'il ne restait à Newton qu'à nous donner une physique aussi exacte que sa géométrie ${ }^{31}$, montre bien toute la difficulté, pour ne pas dire l'impossibilité, pour l'époque, de concevoir une physique purement mathématique et néanmoins complète. On pourrait aussi citer l'opinion de Malebranche qui voyait dans l'usage des mathématiques une “ occasion d'erreur" lorsqu'elles étaient confondues avec la physique ${ }^{\prime 2}$. Cette véritable " conversion des esprits » qu'appelait le modele newtonien, n'est pas aussi sans rappeler, bien qu'elle soit de direction contraire, celle que demandait la " physique céleste » de Kepler. Pour son maître Maëstlin, cette formulation lui paraissait être une contradiction dans les termes et donc une absurdité, tant était encore prégnante la séparation aristotélicienne du ciel et de la terre $^{33}$. Il n'est d'ailleurs pas supertlu de rappeler que Newton lui-même,

28. A. Koyré, Newtonian Studies, Chicago, University of Chicago press, 1968, p. 137.

29. F. Duchesneau, Leibniz et la méthode de la science, op. cit., p. 244-245

30. Ibid., p. 232.

31. I. B. Cohen, Introduction to Newton's Principia, Cambridge, Harvard University Press, 1971, p. 157.

32. E. Ayton, op. cit., p. 113.

33. A. Koyré, La révolution astronomique, Paris, Hermann, 1961, p. 364. 
formé lui aussi dans le cadre du paradigme cartésien, n'a pas complètement abandonné toute tentative d'explication mécanique par contiguité des interactions. Car s'il a su résister à la tentation d'inclure dans les Principia une recherche des causes, les pressions (psychologiques et sociales) exercées sur lui pour fournir une explication mécanique à ce qui semblait à plusieurs de ses contemporains (Leibniz y compris) relever du miracle, l'ont amené, dans sa correspondance et dans des "queries" ajoutées à la deuxième édition de son Opticks (1717), à traiter des causes possibles de la gravitation. Il raviva ainsi la notion d'éther, qui, rappelons-le, dans sa version de support des ondes électromagnétiques, subira d'ailleurs le même sort que les tourbillons de Descartes et de ses successeurs : elle sera à son tour dissoute, au toumant du vingtième siècle, dans les nombreuses contradictions que feront naître les multiples tentatives, infructueuses, de mathématisation. Notons que l'idée newtonienne d'une "physique mathématique " ne s'impose pas d'emblée car elle entraîne avec elle une redéfinition radicale de la notion d'explication. On pourrait en effet montrer que l'abandon de l'explication mécanique dans le domaine de la gravitation n'a pas empêché les physiciens de chercher une telle explication mécanique aux ondes électromagnétiques tout au long du XIX siècle ; la disparition de l'éther est ainsi analogue à l'abandon des tourbillons de Descartes.

On peut lire l'affrontement entre Newton et Leibniz comme un moment important de ce processus de transformation qui mène à l'abandon des causes physiques au profit d'une lecture purement mathématique et fonctionnelle de la nature, "l'explication " physique faisant place à "l'explication " mathématique. Et pour revenir aux préoccupations méthodologiques de Duchesneau, je crois que l'on peut même aller plus loin et suggérer que c'est pour s'être accroché aux principes métaphysiques de continuité et de contiguilté mécanique que Leibniz n'a pu utiliser pleinement son talent de mathématicien (calcul différentiel) et donner ainsi à la nouvelle physique une ouvre du calibre de celle de Newton. Il faudra attendre Varignon et les frères Bernoulli pour voir toute la puissance d'une alliance du paradigme fonctionnaliste de Newton et de l'algorithme leibnizien du calcul différentiel ${ }^{34}$. Ainsi, par une autre de ces ironies dont l'histoire a le secret, les alliés européens de Leibniz contribueront en fait à propager sur le continent une physique newtonienne, c'est-à-dire une physique mathématique qui abandonne la recherche des causes physiques si chères à Leibniz et aux Cartésiens.

Résumons-nous : en abordant d'un cil sympathique les thèses leibniziennes en physique et en méthodologie de la science, Duchesneau nous a donné deux fines études sur la pensée du philosophe allemand. Cependant, il a, à notre humble avis, minimisé les difficultés inhérentes à une conception de la science fondée sur la recherche des causes mécaniques des phénomènes et sur une absolutisation de principes métaphysiques (ou architectoniques) imposés

34. Sur Varignon, voir M. Blay, op. cit. 
a priori qui sont en fait rapidement devenus des obstacles à la conceptualisation mathématique du monde physique. Ce que l'inventeur du calcul différentiel ne semble pas avoir réalisé c'est qu'appliquées aux phénomènes de la nature, les mathématiques qu'il mettait au jour agiraient comme un véritable acide des substances censées rendre raison des phénomènes. Une fois ces substances dissoutes, il ne resterait plus que la structure formelle d'un édifice mathématique. Même Newton, on l'a dit, a eu de la difficulté à accepter complètement cette situation et récemment encore on pouvait entendre un physicien comme David Bohm déplorer lui aussi, comme en écho au Journal des sçavans de 1688 , que la physique soit devenue purement mathématique ${ }^{35}$. Il se faisait peut-être là le prophète d'une éventuelle «science leibnizienne "; chose certaine elle demeure encore aujourd'hui « en marge de la science newtonienne ${ }^{36}$ ». Et, pour paraphraser Fontenelle, j'ajouterais que si «Monsieur Leibniz » n'a pas su rétablir l'explication mécanique des phénomènes, «il n'y a guère apparence qu'elle se relève jamais ${ }^{37}$ ".

\section{Département d'histoire}

Université du Québec à Montréal

35. D. Bohm, Science, Order and Creativity, New York, hantam Books, 1987, p. 610 .

36. F. Duchesneau, Leibniz et la méthode de la science, op. cit., p. 389.

37. Fontenelle, «Eloge de Monsieur Leibniz ", Choix d'éloges de savants, I, mathématiciens-philosophes, Paris, Vrin, 1981, p. 152. Dans ce texte, Fontenelle faisait référence au rétablissement par Leibniz des entéléchies aristotéliciennes. 